\title{
Genome-wide analysis of long noncoding RNA expression profile in nasal mucosa with allergic rhinitis
}

\author{
Xian Wei ${ }^{1 \dagger}$, Man Xu ${ }^{1 \dagger}$, Chao Wang ${ }^{1}$, Shengjian Fang ${ }^{1}$, Yu Zhang ${ }^{1}$ and Weihua Wang ${ }^{1,2^{*}}$
}

\begin{abstract}
Background: Long noncoding RNAs (IncRNAs) are involved in a variety of human immune diseases. However, the expression profile and precise function of IncRNAs in allergic rhinitis (AR) remain unknown. In the present study, genome-wide analysis of IncRNA expression was performed in the nasal mucosa tissue and mRNA regulatory relationship was examined among patients with or without AR.

Methods: Microarray assays were performed and the differential expressions of IncRNAs or mRNA were verified through RT-PCR. The IncRNA functions were annotated using Gene Ontology (GO) and Kyoto Encyclopedia of Genes and Genomes (KEGG). The potential regulatory relationships between IncRNAs and the co-expressed mRNAs were analyzed using Cytoscape software. The expressions of specific IncRNAs and mRNAs were examined using an in vitro cell model.
\end{abstract}

Results: A total of 57 IncRNAs and 127 mRNAs were dysregulated in the nasal mucosa tissue of patients with AR, compared to those of patients without AR (fold change $>2.0$ and $P<0.05$ ). $\mathrm{GO}$ and pathway analysis indicated that the IncRNA-co-expressed mRNAs were enriched in several biological processes and cellular signaling pathways related to AR, such as positive regulation of the integrin biosynthetic process, cell adhesion, and leukocyte transendothelial migration. Some IncRNAs regulated the co-expressed genes in a cis- and/or trans-regulatory manner. Furthermore, allergen exposure significantly increased the expression of Inc-CXCL12-4, CXCL12, and CXCR4 in BEAS-2B cells compared to untreated cells $(P<0.01)$.

Conclusion: The results of the present study suggest that IncRNAs participate in the biological pathways related to AR. Leukocyte transepithelial migration may be a potential target for IncRNAs to regulate allergic inflammation and CXCL12/CXCR4 axis plays an important role in the inflammatory process of AR.

Keywords: Allergic rhinitis, Microarray, Expression profile, Long non-coding RNA, CXCL12, CXCR4

\section{*Correspondence: whwangcn@outlook.com}

${ }^{+}$Xian Wei and Man Xu have contributed equally to this work

1 Department of Otolaryngology-Head and Neck Surgery, Shanghai

East Hospital, Tongji University School of Medicine, 150 Jimo Road,

Shanghai 200120, People's Republic of China

Full list of author information is available at the end of the article

\begin{abstract}
Background
Allergic rhinitis (AR) is an IgE-mediated upper airway inflammatory response, which is becoming a global health problem [1]. AR is characterized by symptoms of congestion, rhinorrhea, sneezing, and itching. Because of the identity of the upper and lower airway allergic inflammatory responses, $40 \%$ of AR patients have or will have asthma [2]. AR seriously affects the quality of life of patients and increases the family and social
\end{abstract}


economic burden [1, 3]. Like other allergic diseases, the etiology of AR has complex components. A growing body of evidence indicates that the imbalance of the Th1/Th2 immune response contributes to the onset of AR [4-6]. However, the underlying pathogenesis of AR remains unclear.

It is well known that most of the genome is transcribed into RNA, but only a very small percentage of the transcripts are protein-coding genes, accounting for only 1.5-2\% [7]. Therefore, there has been a growing interest in the role of noncoding RNAs. Long noncoding RNAs (lncRNAs) are a group of RNA molecules with transcription lengths of more than 200 nucleotides which do not encode any protein products [8]. LncRNAs widely participate in regulatory functions at the epigenetic, transcriptional, and post-transcriptional levels [9]. Although aberrantly expressed lncRNAs have been detected in nasal mucosa with AR in human and animal models [10-12], studies on the roles of lncRNAs are still at a preliminary stage. The expression pattern and function prediction of lncRNAs in AR remain unclear.

The aim of the present study is to examine the expression profiles of lncRNAs and mRNAs in AR. We identified the differential expression of lncRNAs and mRNAs in nasal tissues from $A R$ and non-AR patients using microarray assays. Moreover, we analyzed the potential functions of the differentially expressed lncRNAs via bioinformatics analysis and validated the meaningfully enriched pathway using an in vitro cell culture model.

\section{Materials and methods}

\section{Patients and tissue collection}

A total of 8 AR patients and 10 non-AR patients were admitted to the Department of Otolaryngology-Head and Neck Surgery, Shanghai East Hospital between 2016 and 2019. All of the patients with AR had a positive skin prick test reaction only to dust mites and were diagnosed based on their medical history, nasal endoscopic examination, and allergen skin prick test. None of the participants had received topical or systemic glucocorticoid therapy for 4 weeks before tissue collection. Nasal mucosal tissues were obtained surgically from the inferior turbinates of the patients. The harvested samples were snap-frozen in liquid nitrogen and stored at $-80^{\circ} \mathrm{C}$. All patients had nasal septum deviation and were scheduled to undergo septoplasty and partial removal of the inferior turbinates. The study conforms to the standards of the Declaration of Helsinki. Patients who had a history of previous nasal surgery, smoking, autoimmune diseases, concurrent sinusitis, or systemic diseases were excluded from our study. Patient clinical characteristics are summarized in Additional file 1.

\section{Total RNA extraction}

For the lncRNA and mRNA microarrays, total RNA was extracted from $100 \mathrm{mg}$ of nasal mucosal tissue from 3 AR patients and 3 non-AR patients using TRIzol reagent (Invitrogen, Carlsbad, CA, USA). Total RNA was quantified by a NanoDrop ND-2000 spectrophotometer (Thermo Fisher Scientific, Wilmington, DE, USA) and the RNA integrity was assessed using an Agilent 2100 Bioanalyzer (Agilent Technologies, Santa Clara, CA, USA).

\section{LncRNA chip microarray}

Total RNA labeling, microarray hybridization, and washing were performed using an Affymetrix Human OE IncRNA array (Affymetrix, Santa Clara, CA, USA) based on the manufacturer's instructions. The microarray profiling was conducted in the laboratory of Shanghai OEBiotech (Shanghai, People's Republic of China). This microarray contains probes for 25,986 mRNAs and 66,741 lncRNAs.

\section{Data analysis}

Raw data were extracted using the Affymetrix GeneChip Command Console (version 4.0, Affymetrix). RMA (Robust Multichip Average) normalization for both gene and exon level analysis was performed using Expression Console (version1.3.1, Affymetrix). GeneSpring software (version 13.1, Agilent Technologies) was employed to complete subsequent data processing. After $\log 2$ transformation of the raw signals, differential expression of lncRNAs and mRNAs was defined by the absolute value of fold change $(>2.0)$ and $P$ value $<0.05$ (Student's $t$-test). The unsupervised hierarchical clustering of differentially expressed lncRNAs and mRNAs was carried out. The differentially expressed mRNAs were input into the DAVID database (http://david. abcc.ncifcrf.gov) for Gene Ontology (GO) and Kyoto Encyclopedia of Genes and Genomes (KEGG) pathway annotation classification.

\section{Quantitative RT-PCR validation}

Total RNA was extracted from the nasal mucosal tissue from $8 \mathrm{AR}$ and 10 non-AR patients using TRIzol reagent (Invitrogen) according to the manufacturer's instructions. The first strand cDNA was reverse-transcribed from 500 ng of total RNA using PrimeScript ${ }^{\text {TM }}$ RT Master Mix (Takara Bio, Inc., Otsu, Japan). SYBR Premix Ex Taq ${ }^{\mathrm{TM}}$ (Takara Bio, Inc.) was used to conduct real-time PCR using an ABI 7500 Real-Time PCR System (Applied Biosystems, Foster City, CA, USA). The specific primer sequences used in qRT-PCR are shown in Table 1. The expression levels of lncRNAs and 
Table 1 Primers used for qRT-PCR of IncRNA and mRNA expression

\begin{tabular}{lll}
\hline IncRNA/mRNA & Forward primer $\left(5^{\prime}-3^{\prime}\right)$ & Reverse primer $\left(5^{\prime}-3^{\prime}\right)$ \\
\hline Inc-CBR1-3 & GGTCAAGCCAAGCCAACAG & GTCCTCAGCACCACTTATTAGT \\
Inc-HPR-1 & GAGGCACAGACAGGTTGAGTA & CGGTCACAGCCAAGCAGTA \\
Inc-SEPT7L-5 & CCATGCCATTCAGGTCCAATC & GGAATGGATGCGAATGGAATGA \\
DLGAP1-AS1 & TATCTGAGAGCCAGCGAACTT & CTTCATAGCCTGTTGCGTCAT \\
Inc-CXCL12-4:1 & GACCGCTCCCGCCTAATG & TGCTTAGCCCTCCGGATACC \\
Inc-CXCL12-4:2 & CTACAGATGCCCATGCCGAT & AGGTTGGACACTTGGCTTGT \\
MUC7 & GCTTGCTTCTCGTTCAGT & GTGATGATGCCTTCTGTGA \\
IGFBP3 & GTCCTCCTTAGCACAATGTA & TCCTCCTTCCTGTTCTGATA \\
CXCL12 & GACAAGTGTGCATTGACCCG & GCCCTTCCCTAACACTGGTT \\
CXCR4 & AACTTCAGTTTGTTGGCTGCG & GATCCCCTCCATGGTAACCG \\
GAPDH & TGTTGCCATCAATGACCCCTT & CTCCACGACGTACTCAGCG \\
\hline
\end{tabular}

mRNAs were normalized to glyceraldehyde 3-phosphate dehydrogenase (GAPDH) and quantified using the $2^{-\Delta \Delta \text { ct }}$ method.

\section{Cell culture and allergen treatment}

Human bronchial epithelial cell line BEAS-2B was purchased from ATCC (Walkersville, MD, USA) and cultured in BEGE medium with a Bullet Kit (Lonza, Walkersville, MD, USA). All incubations were carried out in a humidified atmosphere of $5 \% \mathrm{CO}_{2}$ at $37^{\circ} \mathrm{C}$. All experiments were performed with BEAS-2B cells at 80-90\% confluency. Cells were exposed to different concentrations of ovalbumin (OVA) $(100 \mu \mathrm{g} / \mathrm{mL})$ or house dust mite (HDM) (Wolwo Bio-Pharmaceutical, Zhejiang, China) $(40 \mu \mathrm{g} / \mathrm{mL})$ for $4 \mathrm{~h}$.

\section{LncRNA-mRNA co-expression analysis}

Before predicting the possible functions of lncRNAs in AR, a correlation analysis of lncRNAs and mRNAs involved in allergic inflammation was carried out [13]. According to the normalized signal intensity of each differentially expressed lncRNA and mRNA in this microarray assay, Pearson's Correlation Coefficient (PCC) of their expression was calculated to evaluate the correlation between lncRNAs and mRNAs. The co-expressed mRNAs of lncRNAs were identified by $P$ values of $\mathrm{PCC}<0.05$ and absolute values of $\mathrm{PCC}>0.8$.

\section{Functional enrichment analysis of the IncRNAs}

For function prediction of lncRNAs, an enrichment analysis of the co-expressed mRNAs was performed using the hypergeometric cumulative distribution function [13]. The enriched annotations of GO and KEGG pathways were assigned to the corresponding lncRNA as its predicted functions. The threshold of statistical significance was set as $P<0.05$ and false discovery rate
$(\mathrm{FDR})<0.01$. The most enriched annotations reflected the potential functions of the co-expressed lncRNAs.

\section{LncRNA-mRNA regulatory network analysis}

To explore the potential target genes in AR, cis- and trans-regulatory analysis of the differentially expressed lncRNAs was performed. For cis-regulatory analysis, we identified the cis-regulated genes when the coexpressed mRNA loci were within $100 \mathrm{kbp}$ upstream and downstream of the given lncRNA. Another regulatory mechanism of the specific lncRNAs in the expression of certain genes involves the factors mediating chromatin transcription (TFs) $[13,14]$. So for transcriptional factor correlation analysis, the hypergeometric cumulative distribution function was used to compare the co-expressed mRNAs with the genes regulated by certain TFs $(P<0.05$ and FDR $<0.01)$. Then we predicted that these lncRNAs possibly regulated the target genes in a trans-regulatory manner. The lncRNA-TFmRNA network was constructed based on the interactions between the lncRNAs and the co-expressed target mRNAs. We selected the top 10 prediction regulating relationships with the highest prediction reliability to construct the core network map using Cytoscape software.

\section{Statistical analysis}

All data were expressed as mean \pm standard deviation and analyzed using IBM SPSS Statistics, Version 22 (IBM Corp., Armonk, NY, USA). The differences in expression of lncRNAs and mRNAs in nasal mucosal tissue between AR and non-allergic patients were analyzed using Student's $t$-tests. $P<0.05$ was considered statistically significant. 


\section{Results}

\section{Differentially expressed IncRNAs and mRNAs in AR}

We profiled the expression patterns of lncRNAs and mRNAs associated with AR via microarrays. Our data showed that a total of 57 lncRNAs were differentially expressed in the nasal mucosa from AR and non-AR patients, with 22 upregulated and 35 downregulated, as indicated by the volcano plots and heat maps (Fig. 1a, b). Simultaneously, we found that a total of 127 mRNAs were differentially expressed, with 43 mRNAs upregulated and $84 \mathrm{mRNAs}$ downregulated in the nasal mucosa from patients with AR compared to those without AR (Fig. 1c, d) (see Additional file 2 for the differentially expressed mRNAs and lncRNAs). The top 20 differentially expressed lncRNAs and mRNAs are included in Table 2. According to the absolute value of fold change (FC), lnc-MUC7-1 and lnc-AC011294.3.1-6 are the most upregulated and downregulated lncRNAs, respectively. MUC7 and IGFBP3 are the most upregulated and downregulated mRNAs, respectively. These results indicate
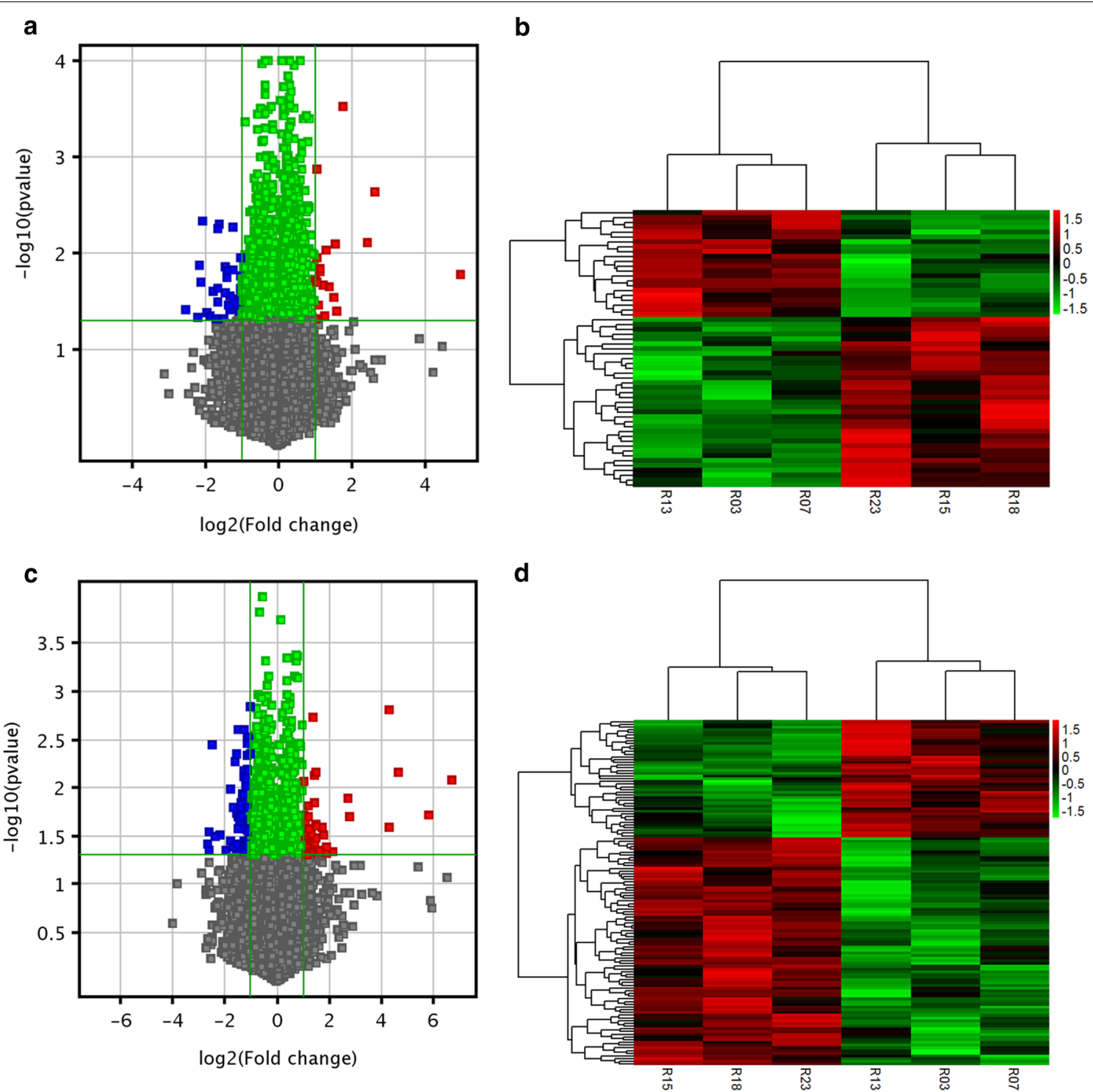

Fig. 1 Differential expression of IncRNAs and mRNAs in the nasal mucosa from AR and non-AR patients. Volcano plots show the differentially expressed IncRNAs (a) and mRNAs (c). The vertical lines correspond to 2.0-fold up and down, and the horizontal lines represent $P$ values $=0.05$. Heat maps indicate hierarchical clustering results of differentially expressed IncRNAs (b) and mRNAs (d). Each row corresponds to one IncRNA or mRNA, and each column represents one sample. Red and green colors indicate upregulated and downregulated expression, respectively. AR samples: R03, R07 and R13; non-AR samples: R15, R18, and R23 
Table 2 Top 20 upregulated and downregulated IncRNAs and mRNAs in the nasal mucosa tissue from patients with AR compared to without AR

\begin{tabular}{|c|c|c|c|c|c|c|c|}
\hline \multicolumn{2}{|c|}{ Upregulated IncRNAs } & \multicolumn{2}{|c|}{ Downregulated IncRNAs } & \multicolumn{2}{|c|}{ Upregulated mRNAs } & \multicolumn{2}{|c|}{ Downregulated mRNAs } \\
\hline IncRNA & FC (abs) & IncRNA & FC (abs) & mRNA & FC (abs) & mRNA & FC (abs) \\
\hline Inc-MUC7-1 & 31.7086 & Inc-AC011294.3.1-6 & 5.8161 & MUC7 & 100.7255 & IGFBP3 & 6.3291 \\
\hline Inc-UMOD-2 & 6.2684 & Inc-SERPINB12-3 & 4.5107 & $\mathrm{HP}$ & 56.9782 & $\mathrm{ROBO} 2$ & 6.0832 \\
\hline Inc-HPR-1 & 5.4470 & Inc-CBR1-3 & 4.3943 & GP2 & 25.1581 & THY1 & 5.9538 \\
\hline Inc-FADD-2 & 3.3699 & Inc-F5-1 & 4.3442 & HPR & 19.7971 & SELP & 5.5017 \\
\hline Inc-AC073416.2-2 & 3.0700 & Inc-TACC2-7 & 4.1595 & IGHD3-10 & 19.2196 & C1QTNF3 & 5.1029 \\
\hline Inc-HTN1-1 & 2.9277 & Inc-XRCC4-6 & 3.8426 & SMR3B & 6.8644 & FMO2 & 4.6298 \\
\hline Inc-STOM-7 & 2.9026 & Inc-TAS2R1-24 & 3.6408 & PIP & 6.4510 & MGARP & 3.8910 \\
\hline Inc-DNAJC3-1 & 2.5966 & Inc-FRG2C-15 & 3.3977 & CA2 & 4.3661 & FAM72C & 3.4443 \\
\hline Inc-ADAM7-1 & 2.4617 & Inc-ADAMTS1-2 & 3.1879 & EFCAB4B & 3.7810 & IER3 & 3.3861 \\
\hline Inc-GPAT2-6 & 2.3813 & Inc-TMEM207-2 & 3.0959 & CRISP2 & 3.6558 & PLXDC1 & 3.2197 \\
\hline Inc-CALM2-10 & 2.3379 & Inc-|G|P-2 & 3.0957 & BHLHA15 & 3.4208 & GPR18 & 3.1555 \\
\hline Inc-CCDC90B-3 & 2.2304 & Inc-FAM185A-3 & 3.0948 & ALDH1L1 & 3.3048 & CCDC169 & 3.0557 \\
\hline Inc-SEPT7L-5 & 2.2265 & Inc-AC023469.1.1-2 & 3.0728 & PAIP2B & 3.0549 & CNTNAP3B & 2.9703 \\
\hline Inc-FDFT1-1 & 2.1435 & Inc-GUCY1A2-1 & 2.7717 & TMEM56 & 2.8856 & GLI3 & 2.9433 \\
\hline Inc-TPPP2-1 & 2.1042 & Inc-SERPINB12-1 & 2.7467 & FCGR3B & 2.8654 & CXCL12 & 2.9255 \\
\hline Inc-GCGR-1 & 2.0608 & Inc-FMO6P-2 & 2.7096 & PYGB & 2.8527 & CLDN1 & 2.8886 \\
\hline Inc-DLG5-2 & 2.0601 & Inc-C10orf68-7 & 2.6243 & ANO5 & 2.8427 & RMI2 & 2.8873 \\
\hline Inc-SLC6A18-1 & 2.0334 & Inc-MARCKS-7 & 2.6001 & C10orf90 & 2.8226 & STON2 & 2.8486 \\
\hline Inc-ATXN7-9 & 2.0315 & Inc-GMPS-5 & 2.5285 & GGTA1P & 2.7631 & EYA1 & 2.8337 \\
\hline Inc-ME1-2 & 2.0308 & DLGAP1-AS1 & 2.5205 & ANO1 & 2.7066 & CAPN5 & 2.8296 \\
\hline
\end{tabular}

FC (abs): absolute fold change

that these lncRNAs and mRNAs may have specific functions in the development of AR.

\section{Validation of the microarray data by qRT-PCR}

To validate the microarray results, we randomly chose 4 lncRNAs and 2 mRNAs from the differentially expressed lncRNAs and mRNAs for qRT-PCR. As Fig. 2 shows, our data indicated that the expression trend of the selected RNAs was consistent with the results of the microarray analysis.

\section{GO and KEGG analysis of the differentially expressed IncRNAs}

There are thousands of co-expression relationships between the differentially expressed IncRNAs and mRNAs. We analyzed the co-expression relationships of the top 500 pairs by PCC and constructed a co-expression network using Cytoscape software (Fig. 3). The visible network also indicated that one lncRNA could regulate the expression of multiple mRNAs, and the expression of the same gene could be regulated by multiple lncRNAs.

The potential functions of the lncRNAs were predicted by the GO and KEGG pathway annotations of their co-expressed mRNAs. The GO categories are biological process, molecular function, and cellular

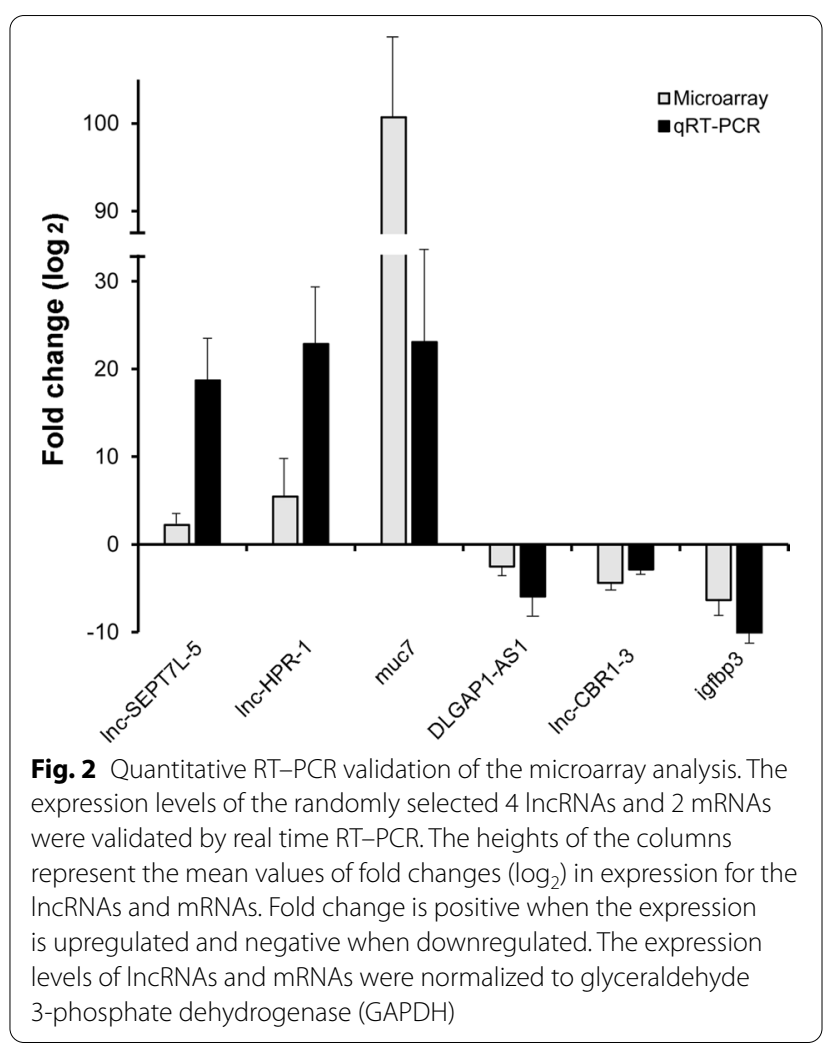




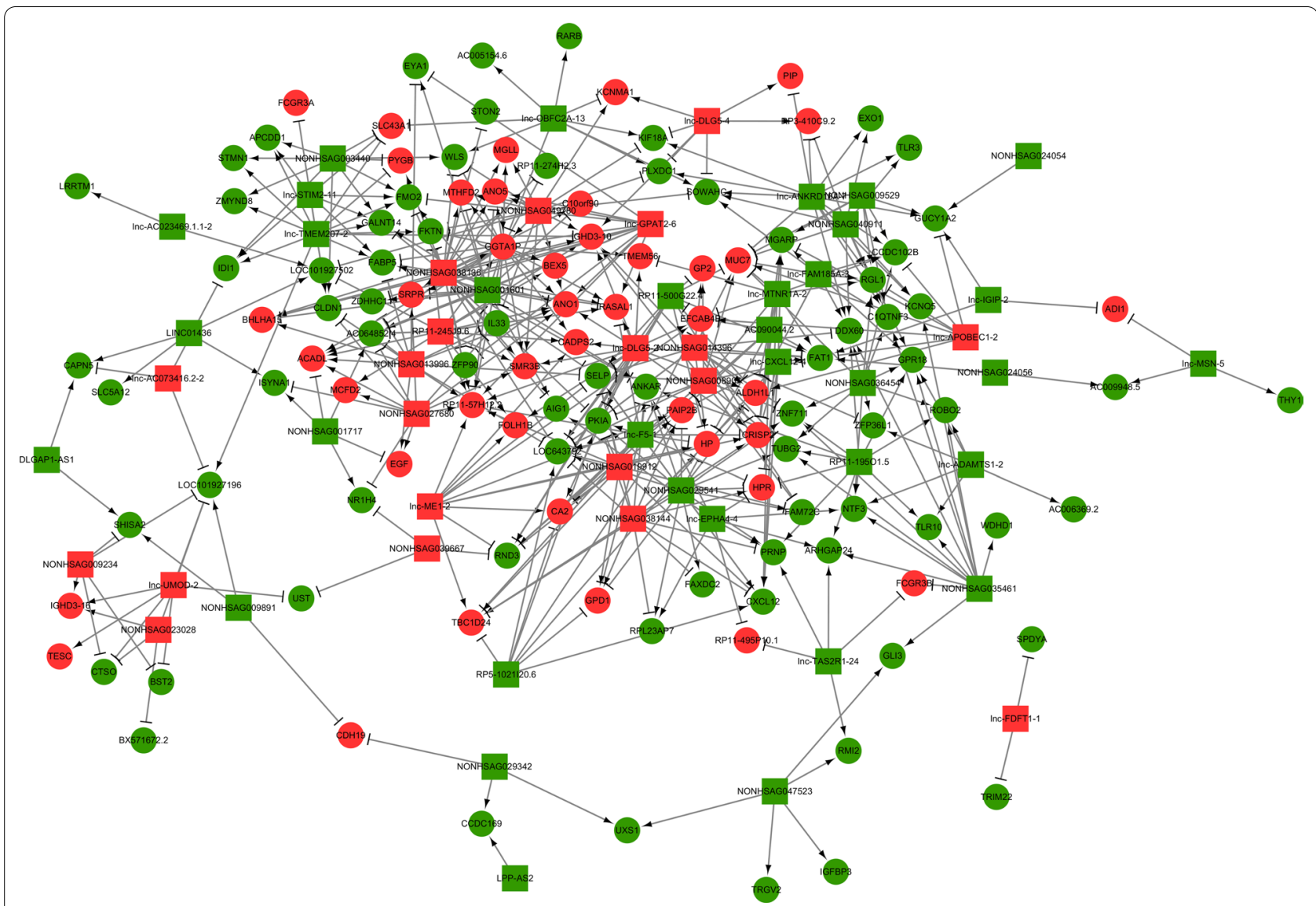

Fig. 3 LncRNA-mRNA co-expression network. The square nodes represent IncRNAs, and the round nodes represent mRNAs. The red and green colors indicate high and low expression, respectively. The lines with arrowheads or blunt ends represent positive or negative regulation, respectively

component. GO analysis of the co-expressed mRNAs revealed that the most enriched annotations were involved in positive regulation of the integrin biosynthetic process, cell adhesion, focal adhesion, inflammatory response, extracellular matrix, $\mathrm{T}$ cell receptor complex, cell junction, and intracellular calcium activated chloride channel activity. We counted and summarized the top $20 \mathrm{GO}$ annotations with the most credentiality (Fig. 4a-c). In addition, the KEGG pathway analysis indicated that protein processing in the endoplasmic reticulum, protein export, the MAPK signaling pathway, and leukocyte transendothelial migration were the most frequently predicted pathways (Fig. 4d). These pathways are associated with immune cell proliferation and migration. The results indicate that these differentially expressed lncRNAs may play important roles in the pathophysiological process of allergic inflammation, such as inflammation, cell differentiation, proliferation, and chemotactic movement.

\section{Analysis of the IncRNA-mRNA regulatory network}

LncRNAs may regulate the nearby genes in a cis-regulatory manner. Therefore, we screened the chromosomal co-expressed mRNAs $100 \mathrm{kbp}$ upstream and downstream of 57 differentially expressed lncRNAs and identified 35 lncRNAs with 41 potential cis-regulated mRNAs. The lncRNAs and the potential cis-regulated mRNAs are included in Table 3.

We calculated the significance of enrichment of each co-expressed mRNA in TFs from the Encyclopedia of DNA Elements, and identified 143 lncRNA-TF pairs, including 50 lncRNAs and 47 TFs. We selected the top 100 lncRNA-TF pairs with the most credentiality and created the IncRNA-TF two-element network relationship using Cytoscape software (Fig. 5a). Adding the above-mentioned co-expressed mRNA, we created the lncRNA-TF-mRNA three-element network relationship. The visible core network map was generated based on the top 10 lncRNA-TF-mRNA pairs (Fig. 5b). As shown in the network map, LPP-AS2 is the regulatory 


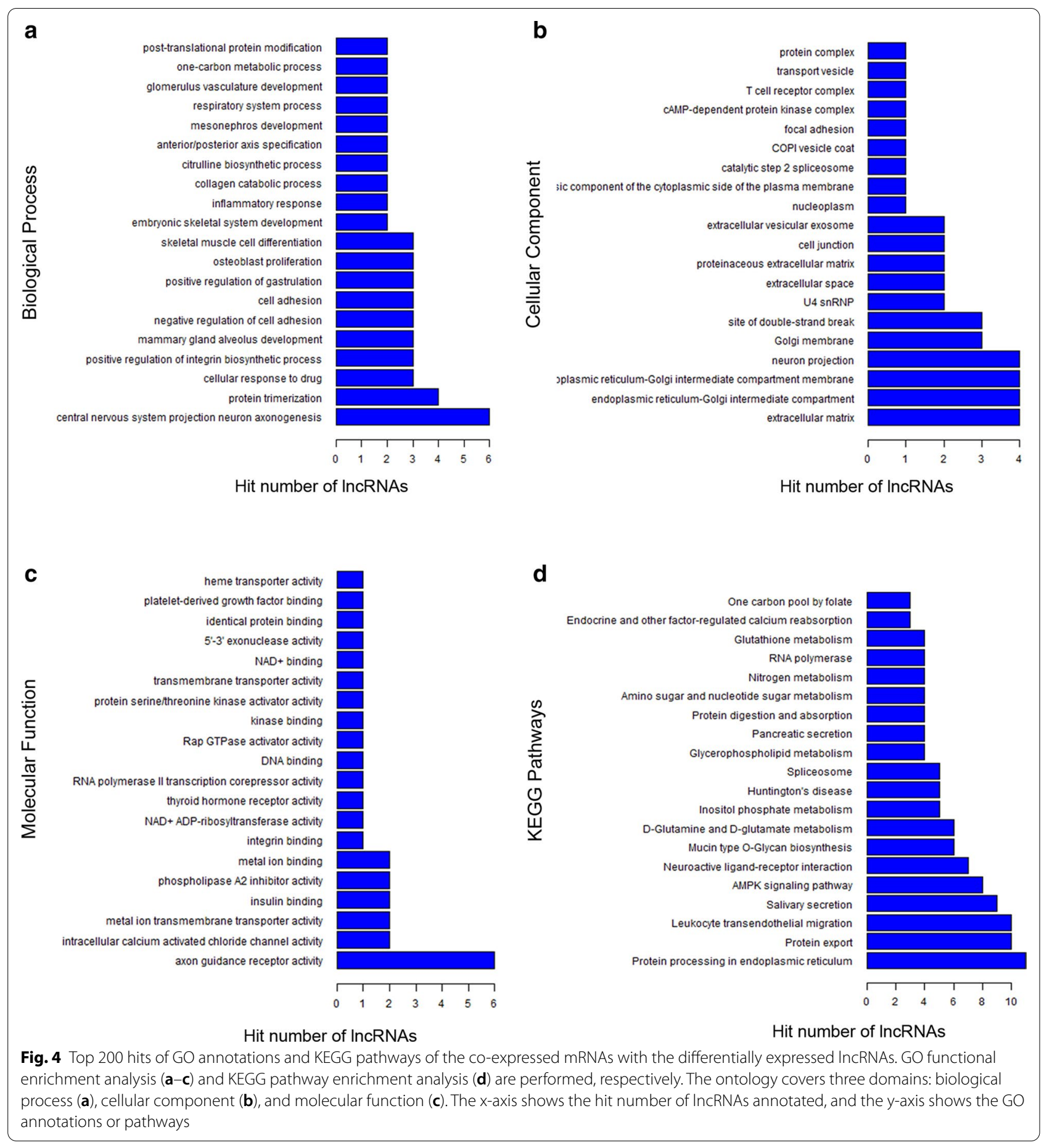

lncRNA with most potential in the trans-regulation of the target mRNAs.

\section{Expression of Inc-CXCL12-4 in airway epithelial cells after allergen stimulation}

Based on the KEGG pathway analysis, we know that leukocyte transendothelial migration is one of the frequently enriched pathways in our functional predictive analysis (Fig. 4d). From the DAVID database, we identified three differentially expressed mRNAs enriched in this pathway, and they are CXCL12 (also known as stromal cell-derived factor-1 $\alpha$, SDF-1 $\alpha$ ), THY1, and CLDN1 (Fig. 6a). The cis-regulatory analysis indicated that lnc-CXCL12-4 and CXCL12 are both from chromosome 10 (Table 3) and 
Table 3 LncRNAs and the potential cis-regulated mRNAs

\begin{tabular}{|c|c|c|c|}
\hline Chrom & Correlation & IncRNA & mRNA \\
\hline 5 & 0.987369 & Inc-XRCC4-6 & VCAN \\
\hline 5 & 0.986823 & Inc-|G|P-2 & CTB-131B5.2 \\
\hline 3 & 0.986734 & Inc-TMEM207-2 & CLDN1 \\
\hline 16 & 0.986412 & Inc-HPR-1 & $\mathrm{HP}$ \\
\hline 2 & 0.983264 & Inc-CALM2-10 & MCFD2 \\
\hline 1 & 0.981938 & Inc-FMO6P-2 & FMO2 \\
\hline 1 & 0.981073 & Inc-F5-1 & SELP \\
\hline 10 & 0.97774 & Inc-DLG5-4 & KCNMA1 \\
\hline 7 & 0.974997 & Inc-FAM185A-3 & LRRC17 \\
\hline 10 & 0.966048 & Inc-CXCL12-4 & CXCL12 \\
\hline 4 & 0.964856 & Inc-MTNR1A-2 & FAT1 \\
\hline 9 & 0.964309 & Inc-STOM-7 & GGTA1P \\
\hline 4 & 0.96054 & Inc-STIM2-11 & $\mathrm{PCDH7}$ \\
\hline 10 & 0.950211 & Inc-C10orf68-7 & C10orf68 \\
\hline 11 & 0.94865 & Inc-GUCY1A2-1 & GUCY1A2 \\
\hline 2 & 0.946103 & Inc-LYPD1-2 & NCKAP5 \\
\hline 16 & 0.945882 & Inc-UMOD-2 & GP2 \\
\hline 16 & 0.944974 & Inc-HPR-1 & HPR \\
\hline 5 & 0.941399 & Inc-TAS2R1-24 & SEMA5A \\
\hline 11 & 0.932664 & Inc-CRTAM-1 & C11orf63 \\
\hline 6 & 0.931226 & Inc-ME1-2 & PGM3 \\
\hline 2 & 0.92093 & Inc-AC023469.1.1-2 & RND3 \\
\hline 4 & 0.917134 & Inc-MUC7-1 & MUC7 \\
\hline- & 0.916819 & Inc-MARCKS-7 & BMS1P6 \\
\hline 21 & 0.910659 & Inc-ADAMTS1-2 & ADAMTS5 \\
\hline 11 & 0.904913 & Inc-FADD-2 & ANO1 \\
\hline 4 & 0.891984 & Inc-MUC7-1 & SMR3B \\
\hline 1 & 0.890773 & Inc-C8B-1 & DAB1 \\
\hline 9 & 0.886791 & Inc-ANKRD18A-7 & CNTNAP3 \\
\hline 2 & 0.88018 & Inc-OBFC2A-13 & MYO1B \\
\hline- & 0.863629 & Inc-MARCKS-7 & RP11-782C8.5 \\
\hline 4 & 0.858795 & Inc-MUC7-1 & SMR3A \\
\hline 2 & 0.856086 & Inc-EPHA4-4 & EPHA4 \\
\hline 1 & 0.837189 & Inc-RAVER2-1 & CACHD1 \\
\hline 4 & 0.834533 & Inc-MUC7-1 & AMBN \\
\hline 13 & 0.829561 & Inc-DNAJC3-1 & DNAJC3 \\
\hline 3 & 0.82015 & Inc-CNTN6-2 & AC090044.2 \\
\hline 21 & -0.8336 & Inc-CBR1-3 & RIMKLBP1 \\
\hline- & -0.85488 & Inc-SEPT7L-5 & BMS1P6 \\
\hline 12 & -0.87456 & Inc-APOBEC1-2 & APOBEC 1 \\
\hline- & -0.88594 & Inc-SEPT7L-5 & RP11-782C8.5 \\
\hline 9 & -0.9127 & Inc-STOM-7 & RP11-477J21.6 \\
\hline
\end{tabular}

lnc-CXCL12-4 may regulate the expression of CXCL12. We established an in vitro experimental environment to mimic the interaction between allergen and airway epithelial barrier. The effects of allergen on the expression of lnc-CXCL12-4 and the related mRNA were evaluated using real-time RT-PCR in BEAS-2B cells. Lower expression levels of lnc-CXCL12-4, CXCL12, and CXCR4 were detected in the unstimulated cells. Four hours after OVA/ HDM exposure, the expression levels of lnc-CXCL12-4, CXCL12, and CXCR4 were significantly increased compared to the untreated cells $(P<0.05)$ (Fig. 6b, c). These results indicate that allergen might induce the expression of lnc-CXCL12-4 at an early stage when allergens enter the airway epithelial barrier, and regulate the signal of the CXCL12/CXCR4 axis in epithelial cells.

\section{Discussion}

LncRNAs are considered to be important regulators of cellular process, such as development, differentiation, and metabolism, through affecting gene expression and cell homeostasis $[15,16]$. Evidence is accumulating that shows lncRNAs are involved in biological functions by interacting with other molecules, such as DNA [17], RNA [18], proteins [19], and metal ions [20]. Abnormal expression of lncRNAs is involved in the pathophysiological process of many diseases, including cancer, respiratory disease, and diabetes [21-23]. Recent studies have explored the expression levels of lncRNAs in upper airway allergic diseases $[5,10,11]$. Ma et al. showed that the expression profile of lncRNAs was altered in the $\mathrm{CD} 4^{+} \mathrm{T}$ cells from AR mice [11]. A change in expression of lncRNAs has been detected in nasal mucosa from patients with AR, but no more bioinformatics analysis was provided [10]. These studies indicate that lncRNAs are involved in the pathogenesis of AR. However, the function and mechanism of action of lncRNAs in AR remain unclear.

In the present study, we assessed genome-wide lncRNA expression patterns in the nasal mucosa from patients with and without AR by microarray analysis, and predicted their possible functions by analyzing the coexpressed mRNAs. Moreover, we also used an in vitro model mimicking the allergen exposure environment of airway epithelium to verify the predicted results. Our results indicated that 57 lncRNA and 127 mRNA transcripts were identified as being differentially expressed between the two groups, including 22 upregulated and 35 downregulated lncRNAs, and 43 upregulated and 84 downregulated mRNAs, respectively. The correlation between some of the differentially expressed mRNAs and AR has been reported, such as ANO1, THY1, CXCL12, and IL33. The expression of ANO1 is higher in AR patients than in healthy controls and hypersecretion of fluid and mucus in AR is closely related to ANO1 [24]. THY1 gene expression was significantly increased in nasal mucosa tissues of AR mice [25]. Expression of CXCL12 in nasal mucosa of seasonal allergic rhinitis patients with asthma was up-regulated predominantly, 

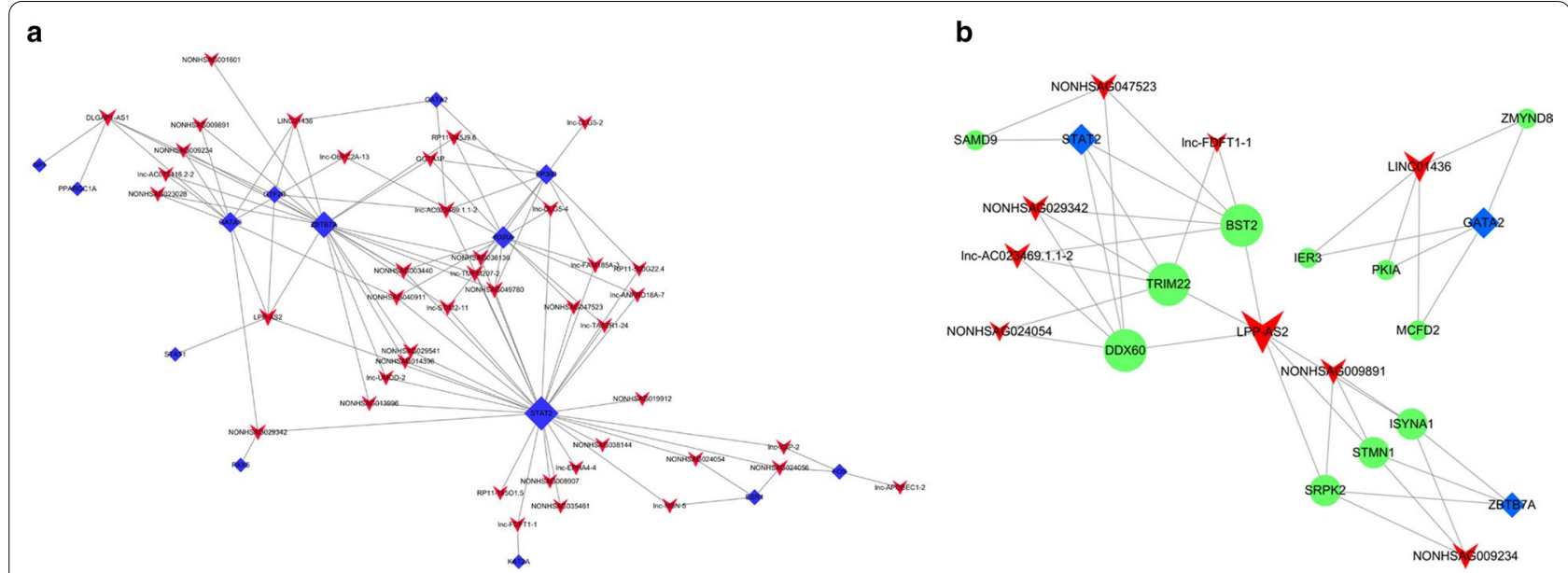

Fig. 5 The core network of trans-regulatory analysis with the differentially expressed IncRNAs. The top 100 IncRNA-transcription factor (TF) pairs with the most credentiality were selected. The IncRNA-TF two-element networks (a) and the IncRNA-TF-mRNA three-element networks (b) are constructed. The red arrowhead nodes represent IncRNAs, the blue rhombus nodes represent TFs, and the green round nodes represent mRNAs

compared with that in seasonal allergic rhinitis patients without asthma [26]. Serum level of IL-33 in patients with AR was significantly higher than in controls and can be used as a marker of the severity of AR [27]. These genes may be involved in the pathophysiological process of AR. To validate the accuracy of microarray analysis, we further randomly chose and validated 4 lncRNAs and 2 mRNAs from the differentially expressed RNAs by qRTPCR. The consistency of our verification results with those of the microarray analysis strongly suggests the reliability of the microarray results.

The functions of the lncRNAs have not yet been fully annotated and the most common method for their functional prediction is through referring to the functional annotations of their co-expressed mRNAs [13]. As shown in Fig. 3, the differentially expressed IncRNAs are coexpressed with hundreds of mRNAs, which may play a vital role in the pathogenesis and development of AR, such as MUC7, IL 33, THY1, and CXCL12. We predicted the functions of the IncRNAs by GO/KEGG enrichment analysis of these co-expressed mRNAs. The most enriched GO annotations are involved in positive regulation of the integrin biosynthetic process, cell adhesion, focal adhesion, inflammatory response, extracellular matrix, T cell receptor complex, cell junction, and intracellular calcium activated chloride channel activity. Some of these functions are known to be involved in the pathogenesis of AR, such as immune cell activation, inflammatory cell migration, and inflammatory response. KEGG pathway analysis also showed that the co-expressed mRNAs regulated some signaling pathways involved in the activity and function of immune cells, including protein processing in the endoplasmic reticulum, protein export, MAPK signaling pathway, and leukocyte transendothelial migration. Interestingly, recent studies have shed light on these biological processes, molecular functions, cellular components, and signaling pathways associated with AR [11, 26, 28, 29].

Due to the variety of functions of lncRNAs, their molecular regulatory mechanism remains unknown [30]. Previous studies have reported that lncRNAs regulate the transcription of nearby genes in a cis-regulatory manner by recruiting remodeling factors to local chromatin [31]. In this study, we explored the cis-regulatory relationships between the differentially expressed lncRNAs and their co-expressed mRNAs (Table 3). We found that the

(See figure on next page.)

Fig. 6 Differentially expressed IncRNAs in airway epithelial cells after allergen stimulation. a Diagram of the Leukocyte Transendothelial Migration pathway [33]. Three dysregulated mRNAs were associated with the Leukocyte Transendothelial Migration pathway in our microarray analysis. They are CXCL12 (also known as stromal cell-derived factor-1a, SDF-1a), THY1, and CLDN1 (shown as CAMs). Their positions in the Leukocyte Transendothelial Migration pathway have been highlighted in yellow. $\mathbf{b}$ Quantitative expressions of Inc-CXCL12-4, CXCL12, and CXCR4 were assessed by real-time RT-PCR from BEAS-2B cells treated with or without $100 \mu \mathrm{g} / \mathrm{mL}$ of OVA for $4 \mathrm{~h}$. c Quantitative expressions of Inc-CXCL12-4 and CXCR4 were assessed by real-time RT-PCR from BEAS-2B cells treated with or without $100 \mu \mathrm{g} / \mathrm{mL}$ of OVA for $4 \mathrm{~h}$. All qRT-PCRs were performed in triplicate, and the $\triangle \mathrm{ct}$ values were calculated by using glyceraldehyde 3-phosphate dehydrogenase as the endogenous control. OVA: ovalbumin; ${ }^{*} P<0.01$ 


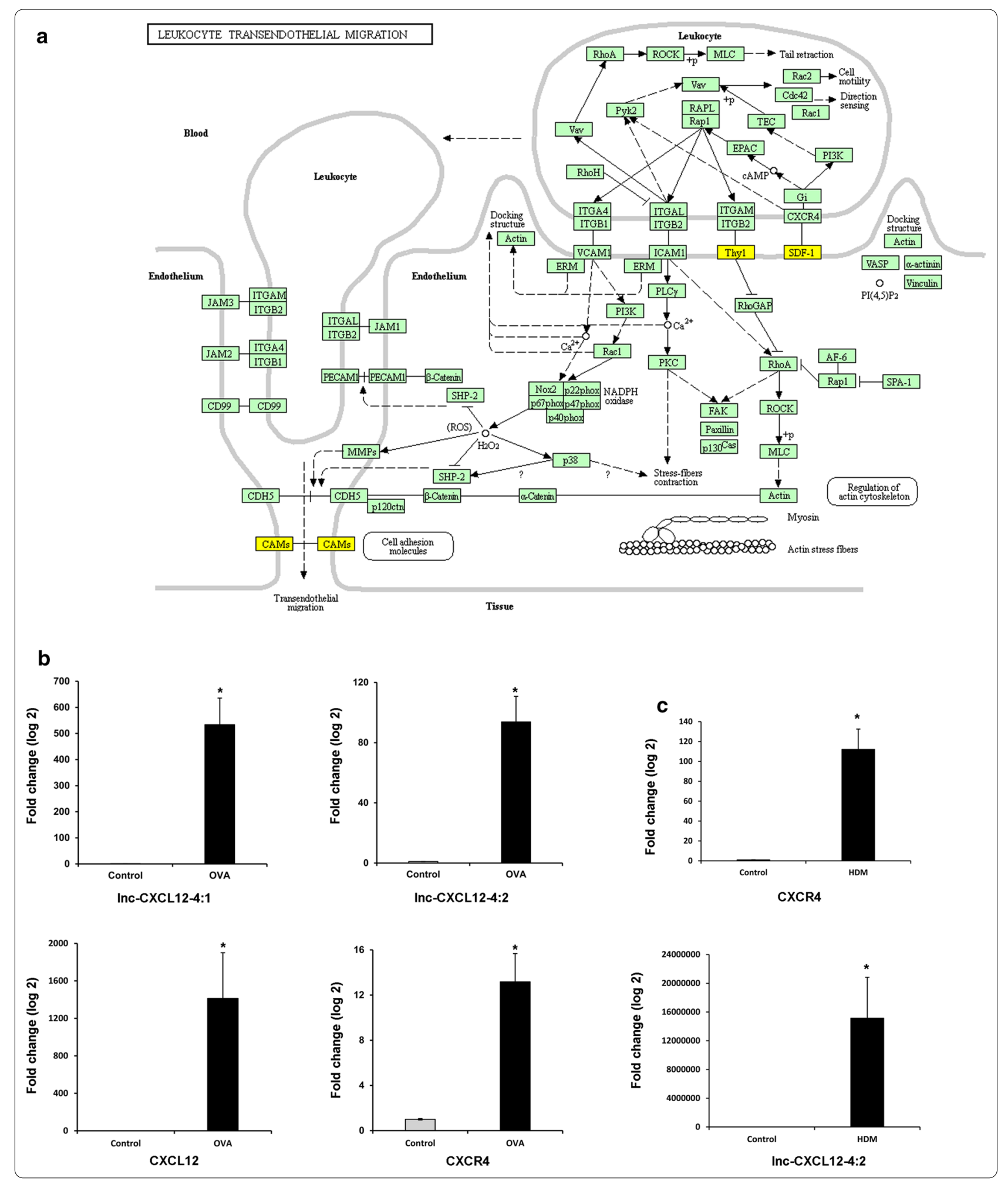


expression of tight junction proteins and chemokines, such as CLDN1 and CXCL12, were cis-regulated by lncTMEM207-2 and lnc-CXCL12-4, respectively. When combined with our KEGG pathway analysis, the differentially expressed CLDN1, CXCL12, and THY1 are involved in leukocyte transendothelial migration. The comprehensive analytical result provides additional information concerning immune cell migration mediated by lncRNAs in the pathogenesis of AR. We also constructed the lncRNA-TF and IncRNA-TF-mRNA network based on the results of trans-regulatory analysis. The core network (Fig. 5) shows that TFs, including STAT2, GATA2, GATA3, and ZBTB7A, regulate lncRNA expression in AR. The expression of SAMD9 is regulated by STAT2, which plays a role in regulating cell proliferation and apoptosis. The proteins encoded by GATA2 and GATA3 play essential roles in regulating the transcription of genes involved in the development and proliferation of hematopoietic cell lineages and $\mathrm{T}$ cells. Diseases associated with ZBTB7A include photosensitive epilepsy and lymphoma. Thus, trans-regulatory analysis provides another way to predict the functions of lncRNAs in the pathogenesis of AR.

Besides genetic and lifestyle-related factors, AR is also affected by the composition of inhaled air. The respiratory epithelial cells may mediate parts of the innate and adaptive immunity by their antigen presentation, phagocytosis, cytokine secretion, and pattern recognition abilities [32]. The epithelial surface of the respiratory tract is the "first battlefield" of allergic inflammation, where the epithelial cells interact with the inhaled allergens and trigger inflammatory cascade reactions. A recent study found that CXCL12 and the chemokine receptor CXCR4 were critical components of the inflammatory processes involved in a murine model of allergic airway disease [26]. Upon interaction with CXCR4, CXCL12 can result in the most efficacious chemoattraction of $\mathrm{T}$ lymphocytes. In the present study, we examined the epithelial responses to allergen exposure using a cell culture model and demonstrated that OVA/HDM exposure induced the expression of lnc-CXCL12-4, CXCL12, and CXCR4 in BEAS-2B within a short time after exposure compared to untreated cells. This is consistent with our previous clinical observations. In nasal polyps from patients with AR, the expressions of lnc-CXCL12-4, CXCL12, and CXCR4 were increased significantly compared to those from nasal polyps without AR. Taken together, these data support the potential importance of lnc-CXCL12-4 and the CXCL12/CXCR4 axis in the immune responses and inflammation in AR.

\section{Conclusions}

A series of aberrantly expressed lncRNAs may participate in the regulation of target protein-coding genes involved in the biological pathways related to AR in cis- and transregulatory manners. On the basis of these findings, we propose that the CXCL12/CXCR4 axis plays a very significant role in the inflammatory process of $\mathrm{AR}$, which is regulated by lnc-CXCL12-4. Leukocyte transepithelial migration may be a potential target for lncRNAs to regulate allergic inflammation.

\section{Abbreviations \\ AR: Allergic rhinitis; FC: Fold change; FDR: False discovery rate; GO: Gene ontology; HDM: House dust mite; KEGG: Kyoto Encyclopedia of Genes and Genomes; LncRNAs: Long noncoding RNAs; OVA: Ovalbumin; PCC: Pearson's Correlation Coefficient.}

\section{Supplementary Information}

The online version contains supplementary material available at https://doi. org/10.1186/s12920-021-00949-4.

Additional file 1: Table S1. Clinical characteristics of AR and non-AR patients.

Additional file 2: Table S2. The differentially expressed mRNAs and IncRNAs.

\section{Acknowledgements \\ The authors thank all the patients who agreed to participate in the study.}

\section{Authors' contributions}

XW: collection and/or assembly of data, manuscript writing, and data analysis; MX: manuscript writing, data analysis and cell experiment. CW, SF, and YZ: collection and/or assembly of data; WW: concept and design, figures preparation, data analysis, manuscript writing, and final approval of manuscript.

\section{Funding}

This work was funded by Reserve Personnel Training Program of Shanghai East Hospital (2019YHRCJH02) and Health Commission of Jiangxi Province Science \& Technology Program (20194085).

\section{Availability of data and materials}

The datasets generated and/or analysed during the current study are available in NCBI Gene Expression Omnibus, http://www.ncbi.nlm.nih.gov/geo, GEO Submission: GSE159415.

\section{Declarations}

\section{Ethics approval and consent to participate}

The study conforms to the standards of the Declaration of Helsinki. This study was approved by the Ethics Committee of Shanghai East Hospital (Permit Number: 2016033). Written informed consent was obtained from all participating patients.

\section{Consent for publication}

Not applicable.

Competing interests

The authors declare that they have no competing interests. 


\section{Author details}

${ }^{1}$ Department of Otolaryngology-Head and Neck Surgery, Shanghai East Hospital, Tongji University School of Medicine, 150 Jimo Road, Shanghai 200120, People's Republic of China. ${ }^{2}$ Department of Otolaryngology-Head and Neck Surgery, Shanghai East Hospital Ji'an Hospital, Ji'an, Jiangxi, People's Republic of China.

Received: 7 January 2021 Accepted: 16 March 2021

Published online: 09 April 2021

\section{References}

1. Cheng L, Chen J, Fu Q, He S, Li H, Liu Z, et al. Chinese society of allergy guidelines for diagnosis and treatment of allergic rhinitis. Allergy Asthma Immunol Res. 2018;10(4):300-53.

2. Wheatley LM, Togias A. Clinical practice. Allergic rhinitis N Engl J Med. 2015:372(5):456-63

3. Meltzer EO. Allergic rhinitis: burden of illness, quality of life, comorbidities, and control. Immunol Allergy Clin N Am. 2016;36(2):235-48.

4. Meng Q, Li P, Li Y, Chen J, Wang L, He L, et al. Broncho-vaxom alleviates persistent allergic rhinitis in patients by improving Th1/Th2 cytokine balance of nasal mucosa. Rhinology. 2019;57(6):451-9.

5. Zhu X, Wang X, Wang Y, Zhao Y. Exosomal long non-coding RNA GAS5 suppresses Th1 differentiation and promotes Th2 differentiation via downregulating $\mathrm{EZH} 2$ and T-bet in allergic rhinitis. Mol Immunol. 2020;118:30-9.

6. Wang Y, Sha J, Wang H, An L, Liu T, Li L. P-FN12, an H4R-based epitope vaccine screened by phage display, regulates the Th1/Th2 balance in rat allergic rhinitis. Mol Ther Methods Clin Dev. 2018;11:83-91.

7. Alexander RP, Fang G, Rozowsky J, Snyder M, Gerstein MB. Annotating non-coding regions of the genome. Nat Rev Genet. 2010;11(8):559-71.

8. Fatica A, Bozzoni I. Long non-coding RNAs: new players in cell differentiation and development. Nat Rev Genet. 2014;15(1):7-21.

9. Kung JT, Colognori D, Lee JT. Long noncoding RNAs: past, present, and future. Genetics. 2013;193(3):651-69.

10. Ma Z, Teng Y, Liu X, Li J, Mo J, Sha M, et al. Identification and functional profiling of differentially expressed long non-coding RNAs in nasal mucosa with allergic rhinitis. Tohoku J Exp Med. 2017;242(2):143-50.

11. Ma Y, Shi L, Zheng C. Microarray analysis of IncRNA and mRNA expression profiles in mice with allergic rhinitis. Int J Pediatr Otorhinolaryngol. 2018:104:58-65.

12. Qian X, Shi S, Zhang G. Long non-coding RNA antisense non-coding RNA in the INK4 locus expression correlates with increased disease risk, severity, and inflammation of allergic rhinitis. Medicine (Baltimore). 2019:98(20):e15247.

13. Guttman M, Amit I, Garber M, French C, Lin MF, Feldser D, et al. Chromatin signature reveals over a thousand highly conserved large non-coding RNAs in mammals. Nature. 2009;458(7235):223-7.

14. Guttman M, Donaghey J, Carey BW, Garber M, Grenier JK, Munson G, et al lincRNAs act in the circuitry controlling pluripotency and differentiation. Nature. 2011;477(7364):295-300.

15. Luo M, Li Z, Wang W, Zeng Y, Liu Z, Qiu J. Long non-coding RNA H19 increases bladder cancer metastasis by associating with EZH2 and inhibiting E-cadherin expression. Cancer Lett. 2013;333(2):213-21.
16. Spurlock CF 3rd, Crooke PS 3rd, Aune TM. Biogenesis and transcriptional regulation of long noncoding RNAs in the human immune system. J Immunol. 2016;197(12):4509-17.

17. Villegas VE, Zaphiropoulos PG. Neighboring gene regulation by antisense long non-coding RNAs. Int J Mol Sci. 2015;16(2):3251-66.

18. Hu S, Wang $X$, Shan $G$. Insertion of an Alu element in a IncRNA leads to primate-specific modulation of alternative splicing. Nat Struct Mol Biol. 2016;23(11):1011-9.

19. Warburton AJ, Boone DN. Insights from global analyses of long noncoding RNAs in breast cancer. Curr Pathobiol Rep. 2017;5(1):23-34.

20. Nelson BR, Makarewich CA, Anderson DM, Winders BR, Troupes CD, Wu F, et al. A peptide encoded by a transcript annotated as long noncoding RNA enhances SERCA activity in muscle. Science. 2016;351(6270):271-5.

21. Wang Y, Qian CY, Li XP, Zhang Y, He H, Wang J, et al. Genome-scale long noncoding RNA expression pattern in squamous cell lung cancer. Sci Rep. 2015:5:11671.

22. Zhang J, Zhu Y, Wang R. Long noncoding RNAs in respiratory diseases. Histol Histopathol. 2018;33(8):747-56.

23. Shan K, Liu C, Liu BH, Chen X, Dong R, Liu X, et al. Circular noncoding RNA HIPK3 mediates retinal vascular dysfunction in diabetes mellitus. Circulation. 2017;136(17):1629-42.

24. Kim HJ, Nam YR, Nam JH. Flos magnoliae inhibits chloride secretion via ANO1 inhibition in calu-3 cells. Am J Chin Med. 2018:46(5):1079-92.

25. Liu Z, Yang X, Liu X, Mu Y, Wang L, Song X, et al. Analysis of expression of ILC2 cells in nasal mucosa based on animal model of allergic bacterial infection rhinitis. J Infect Public Health. 2021;14(1):77-83.

26. Gonzalo JA, Lloyd CM, Peled A, Delaney T, Coyle AJ, Gutierrez-Ramos JC Critical involvement of the chemotactic axis CXCR4/stromal cell-derived factor-1 alpha in the inflammatory component of allergic airway disease. J Immunol. 2000;165(1):499-508.

27. Gluck J, Rymarczyk B, Rogala B. Serum IL-33 but not ST2 level is elevated in intermittent allergic rhinitis and is a marker of the disease severity. Inflamm Res Off J Eur Histamine Res Soc. 2012;61(6):547-50.

28. Aune TM, Spurlock CF 3rd. Long non-coding RNAs in innate and adaptive immunity. Virus Res. 2016;212:146-60.

29. Hu G, Tang Q, Sharma S, Yu F, Escobar TM, Muljo SA, et al. Expression and regulation of intergenic long noncoding RNAs during T cell development and differentiation. Nat Immunol. 2013:14(11):1190-8.

30. Guttman M, Rinn JL. Modular regulatory principles of large non-coding RNAs. Nature. 2012:482(7385):339-46.

31. Guenzl PM, Barlow DP. Macro IncRNAs: a new layer of cis-regulatory information in the mammalian genome. RNA Biol. 2012:9(6):731-41.

32. Toppila-Salmi S, van Drunen CM, Fokkens WJ, Golebski K, Mattila P, Joenvaara S, et al. Molecular mechanisms of nasal epithelium in rhinitis and rhinosinusitis. Curr Allergy Asthma Rep. 2015;15(2):495.

33. Kanehisa M, Furumichi M, Sato $Y$, Ishiguro-Watanabe $M$, Tanabe $M$. KEGG: integrating viruses and cellular organisms. Nucleic Acids Res. 2021;49(D1):D545-51.

\section{Publisher's Note}

Springer Nature remains neutral with regard to jurisdictional claims in published maps and institutional affiliations.

Ready to submit your research? Choose BMC and benefit from

- fast, convenient online submission

- thorough peer review by experienced researchers in your field

- rapid publication on acceptance

- support for research data, including large and complex data types

- gold Open Access which fosters wider collaboration and increased citations

- maximum visibility for your research: over 100M website views per year

At $\mathrm{BMC}$, research is always in progress.

Learn more biomedcentral.com/submissions 